\title{
Effect of hyperfine-induced spin mixing on the defect-enabled spin blockade and spin filtering in GaNAs
}

\author{
Yuttapoom Puttisong, X J. Wang, Irina Buyanova and Weimin Chen
}

\section{Linköping University Post Print}

N.B.: When citing this work, cite the original article.

Original Publication:

Yuttapoom Puttisong, X J. Wang, Irina Buyanova and Weimin Chen, Effect of hyperfineinduced spin mixing on the defect-enabled spin blockade and spin filtering in GaNAs, 2013, Physical Review B. Condensed Matter and Materials Physics, (87), 12.

http://dx.doi.org/10.1103/PhysRevB.87.125202

Copyright: American Physical Society http://www.aps.org/ 


\title{
Effect of hyperfine-induced spin mixing on the defect-enabled spin blockade and spin filtering in GaNAs
}

\author{
Y. Puttisong, ${ }^{1}$ X. J. Wang, ${ }^{1,2}$ I. A. Buyanova, ${ }^{1}$ and W. M. Chen ${ }^{1}$ \\ ${ }^{1}$ Department of Physics, Chemistry and Biology, Linköping University, S-581 83 Linköping, Sweden \\ ${ }^{2}$ National Laboratory for Infrared Physics, Shanghai Institute of Technical Physics, Chinese Academy of Sciences, \\ 200083 Shanghai, People's Republic of China
}

(Received 15 November 2012; revised manuscript received 14 February 2013; published 8 March 2013)

\begin{abstract}
The effect of hyperfine interaction (HFI) on the recently discovered room-temperature defect-enabled spinfiltering effect in GaNAs alloys is investigated both experimentally and theoretically based on a spin Hamiltonian analysis. We provide direct experimental evidence that the HFI between the electron and nuclear spin of the central $\mathrm{Ga}$ atom of the spin-filtering defect, namely, the $\mathrm{Ga}_{i}$ interstitials, causes strong mixing of the electron spin states of the defect, thereby degrading the efficiency of the spin-filtering effect. We also show that the HFI-induced spin mixing can be suppressed by an application of a longitudinal magnetic field such that the electronic Zeeman interaction overcomes the HFI, leading to well-defined electron spin states beneficial to the spin-filtering effect. The results provide a guideline for further optimization of the defect-engineered spin-filtering effect.
\end{abstract}

DOI: 10.1103/PhysRevB.87.125202

PACS number(s): 72.25.Fe, 71.70.Jp, 78.20.Ls, 76.70.Hb

\section{INTRODUCTION}

Understanding spin interaction and spin-coupling mechanisms is an essential key to manipulation of spin properties in materials and to a practical control of spin functionality in spin-based electronic/photonic devices for future spintronics and information processing. ${ }^{1-3}$ In a semiconductor system, hyperfine interaction (HFI) between a confined electron spin and local nuclear spin(s) represents an important spin interaction mechanism. ${ }^{4-7}$ It is well known that HFI can promote electron-nuclear (e-n) spin transfer via mutual e-n spin flip-flops, providing a means for quantum manipulation of an e-n spin system. ${ }^{8,9}$ On the other hand, HFI can also mix spin states and leads to electron and nuclear spin relaxation and decoherence. ${ }^{10-14}$ HFI has been shown to be of paramount importance in a large variety of semiconductor systems that are promising for future spintronics and spin-based quantum computation. Examples include its vital role in the spin blockade in semiconductor double quantum dots (QDs) due to the mixing of singlet/triplet states, ${ }^{10,11}$ in the magnetoresistance effect observed in organic semiconductors, ${ }^{15}$ in spin decoherence/relaxation in InAs QDs, ${ }^{16}$ in hyperpolarization of local nuclear spins in semiconductors, ${ }^{17}$ and in controlling electron spin coherence time of the $\mathrm{P}$ donor site in $\mathrm{Si}^{6}$ and the nitrogen-vacancy center in diamond in the vicinity of ${ }^{13} \mathrm{C}$ nuclei spin bath. ${ }^{7}$

In this work, we attempt to examine the role of HFI in the room-temperature (RT) defect-engineered spin-filtering effect, which has recently been demonstrated to be able to generate $>40 \%$ spin polarization $\left(P_{e}\right)$ of free electrons ${ }^{18-28}$ and to amplify fast-modulating spin signals up to $2700 \%$ at $\mathrm{RT}^{29}$ in $\mathrm{Ga}$ (In)NAs alloys. Since the key to the success of the defect-engineered spin-filtering is generation and maintaining of strong spin polarization of the electron localized at the spin-filtering defects, namely, $\mathrm{Ga}_{i}^{2+}$ interstitials, ${ }^{18,22-25}$ it is natural to ask whether HFI-induced spin mixing at such defects with a nonzero nuclear spin $(I=3 / 2$ for its core $\mathrm{Ga}$ atom) will significantly affect the spin-filtering effect. We shall demonstrate that HFI indeed partially lifts the spin blockade of the spin-dependent recombination (SDR) via the $\mathrm{Ga}_{i}^{2+}$ defects and thus degrades the spin-filtering ability, due to strong e-n spin mixing at the defects. A detailed spin Hamiltonian analysis of the e-n spin coupling provides us with a better understanding of the spin interactions and also a guideline for optimization of the spin-filtering effect.

\section{EXPERIMENTAL}

A large set of GaNAs samples, consisting of both epilayers and multiple-quantum wells (MQWs), were studied. They were grown at $420-580{ }^{\circ} \mathrm{C}$ by molecular-beam epitaxy (MBE) on (001) GaAs substrates. The thickness of the epilayers is about $100 \mathrm{~nm}$. The MQWs are seven periods of 3to 7-nm-thick GaNAs layers sandwiched between 20.2-nmthick GaAs barrier layers. All studied samples exhibit a strong RT spin-filtering effect due to the introduction of the $\mathrm{Ga}_{i}$ interstitials. ${ }^{18,22-25}$ We chose contactless magneto-optical spectroscopy to study the effect of HFI on the spin filtering to avoid unknown magnetoresistance effects and poor electrical contacts that may be present in electrical measurements. The specific magneto-optical spectroscopy employed in this work was optical orientation in an applied longitudinal magnetic field carried out at RT in a Faraday geometry, in which the optical excitation beam was propagating along the magnetic field direction. A Ti-sapphire laser was used as an excitation source with an excitation wavelength $\sim 910-930 \mathrm{~nm}$. Circular polarization of the excitation light was produced by using a $\lambda / 4$ wave retarder in a conjunction of a linear polarizer. Photoluminescence (PL) emissions were guided through a monochromator and were registered by a liquid-nitrogencooled germanium detector. To further provide evidence for spin mixing induced by HFI, optically detected magnetic resonance (ODMR) was used with a microwave frequency (MW) in the X-band range $(\sim 9.2 \mathrm{GHz})$. ODMR was performed at $3 \mathrm{~K}$ under linearly polarized excitation at $900 \mathrm{~nm}$ to single out the HFI effect without possible influence of dynamic nuclear polarization (DNP) of the $\mathrm{Ga}_{i}$ interstitials. ODMR experiments 
(a) Evidence for spin blockade/filtering
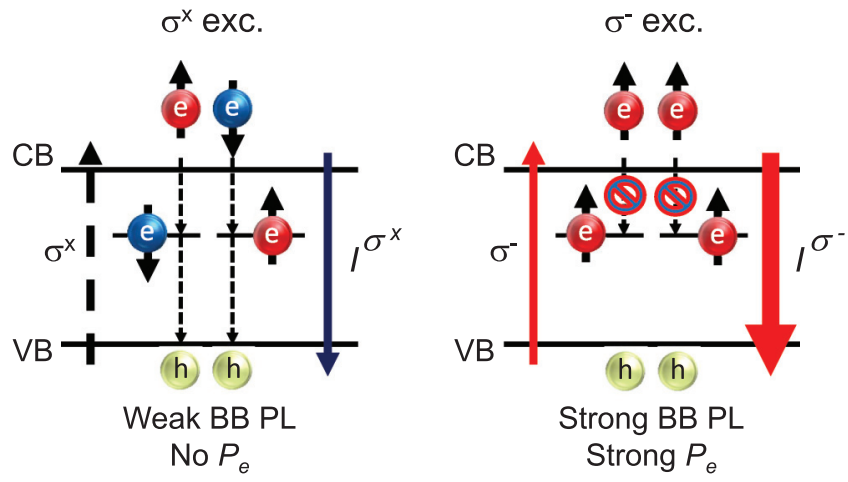

(b) $\mathrm{HFI}$ effect on spin blockade/filtering
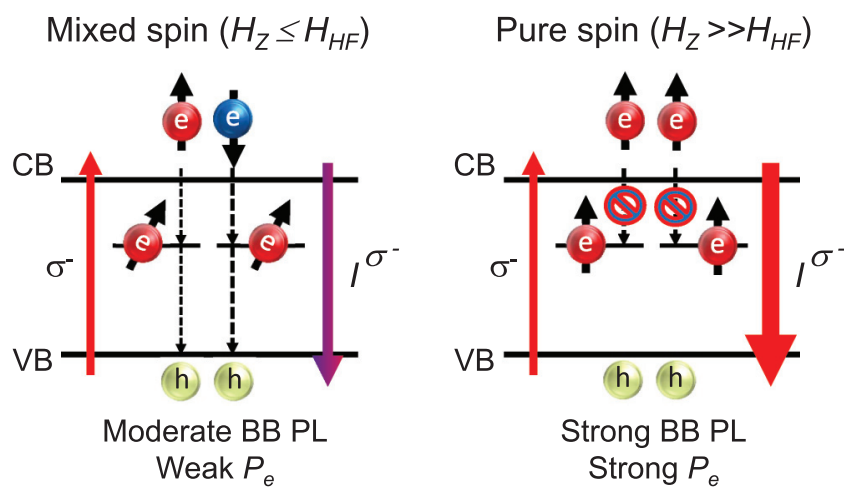

FIG. 1. (Color online) (a) Evidence for the spin-blockade and spin-filtering effect under optical orientation. (b) Effect of hyperfineinduced electron spin mixing (symbolized by the tilted spins) on the spin-blockade and spin-filtering effect. The spin of photoexcited holes is unpolarized due to fast spin relaxation.

at a higher MW frequency in the Q-band range (34 GHz), and thus in higher magnetic fields, were also undertaken for comparison.

\section{RESULTS AND DISCUSSION}

The defect-engineered spin-filtering effect can be activated (or deactivated) under circularly (or linearly) polarized optical excitation in optical orientation experiments ${ }^{18}$ [see Fig. 1(a)], by taking advantage of the electric-dipole selection rule for the band-to-band (BB) optical transition of the GaNAs alloy. ${ }^{30}$ Under linearly polarized excitation $\left(\sigma^{x}\right)$, no electron-spin polarization of the conduction band (CB) and the $\mathrm{Ga}_{i}^{2+}$ defects can be created. Random and equally probable capture and recombination of both spin-up and spin-down CB electrons via the defects with a randomly oriented electron spin result in weak BB PL and zero $P_{e}$; see the left panel of Fig. 1(a). On the contrary, circularly polarized excitation can align $\mathrm{CB}$ electron spins ${ }^{30}$ and also the defect electron through a dynamic polarization process promoted by SDR. ${ }^{18-29}$ Once the electron at the defect is spin polarized, as illustrated in the right panel of Fig. 1(a), assuming complete spin polarization of the defect electron $\left(P_{c}=100 \%\right)$, the spin blockade will prevent further capture and recombination of the photogenerated CB electrons by the defects. This leads to strong $P_{e}$ as well as an increase in the BB PL intensity as compared with that under $\sigma^{x}$ excitation. Whenever a $\mathrm{CB}$ electron undergoes a spin flip, it will immediately be captured and spin filtered out by the defects thanks to a longer spin lifetime of the defect electron such that strong $P_{e}$ can be maintained. ${ }^{18-29}$ This is ensured by a very short capture time of $\mathrm{CB}$ electrons by the defects, i.e., $\tau_{C}<10 \mathrm{ps}$, which is more than one order of magnitude faster than their spin relaxation time $\tau_{S} \sim 100 \mathrm{ps}^{20,21}$ Therefore, the extent of the spin-blockade and spin-filtering effect can be evaluated by $P_{e}$ or the ratio between the BB PL intensity under circular and linear excitation-the so-called SDR ratio $\left(I^{\sigma^{-}} / I^{\sigma^{x}}\right.$ or $\left.I^{\sigma^{+}} / I^{\sigma^{x}}\right)$. The absence and presence of the blockade and filtering effect are characterized by $I^{\sigma^{-}} / I^{\sigma^{x}}=I^{\sigma^{+}} / I^{\sigma^{x}}=1$ and $I^{\sigma^{-}} / I^{\sigma^{x}}>1$ (or $I^{\sigma^{+}} / I^{\sigma^{x}}>1$ ), respectively.

When the spin-filtering defect core $\mathrm{Ga}_{i}^{2+}$ contains a nonzero nuclear spin, i.e., $I=3 / 2$ for both naturally abundant isotopes ${ }^{69} \mathrm{Ga}_{i}$ and ${ }^{71} \mathrm{Ga}_{i}$, a nonvanishing HFI is expected to lead to mixing of electron spin states. ${ }^{14}$ As depicted in the left panel of Fig. 1(b), such mixing lifts the spin blockade such that the defects can capture $\mathrm{CB}$ electrons of both spin orientations. The values of $P_{e}$ or SDR ratio critically depend on the extent of the spin mixing. Application of a longitudinal magnetic field $\left(B_{z}\right)$ is expected to suppress the HFI-induced spin mixing and to recover the spin-blockade and spin-filtering functionality of the defects, as illustrated in the right panel of Fig. 1(b), when the Zeeman interaction of the defect electron $\left(H_{Z}\right)$ in high fields overcomes the field-independent $\mathrm{HFI}\left(H_{\mathrm{HF}}\right)$.

To examine the influence of the HFI on the spin blockade and filtering, we measured the SDR ratio as a function of $B_{z}$. The same conclusion could be drawn by monitoring $P_{e}$, not shown here. A representative experimental curve is displayed in Fig. 2(a), taking as an example the 7-nm-thick $\mathrm{GaN}_{0.018} \mathrm{As}_{0.082} \mathrm{MQWs}$. It clearly shows the evidence of the spin-blockade and filtering, yielding an SDR ratio of 1.1. With increasing magnetic field strength in either direction, a sizable increase of the SDR ratio can be observed that saturates at $I^{\sigma^{-}} / I^{\sigma^{x}} \approx 1.17$ when $\left|B_{z}\right|>4000 \mathrm{G}$. (The absolute value of the SDR ratio can vary between samples, as it depends on many parameters such as defect concentrations. However, the physics of the concerned SDR effect remains the same in all studied samples.) Here, we have purposely chosen very weak optical excitation density such that DNP is negligible and the spin mixing effect can be singled out. Under this condition, we have also ensured that the lifetime is much longer than the spin relaxation time for the bound electron at the $\mathrm{Ga}_{i}^{2+}$ defect, which was confirmed from our Hanle studies conducted in a transverse magnetic field. In this case, the spin of the defect electron has nearly relaxed to the full extent at $B_{z} \approx 0$ when the time-independent quantum-mechanical treatments (to be presented below) provide the best description of the energies and states of the coupled e-n spin system. Similar field dependence of the SDR ratio was also reported in a recent study, which was phenomenologically described by the magnetic field suppression of spin relaxation of bound electrons. ${ }^{28}$ The exact physical mechanism responsible for the observation remains unresolved so far, however, due to a lack of direct experimental evidence.

To examine if the observed field-induced enhancement of the spin-blockade and spin-filtering effect is indeed caused by 


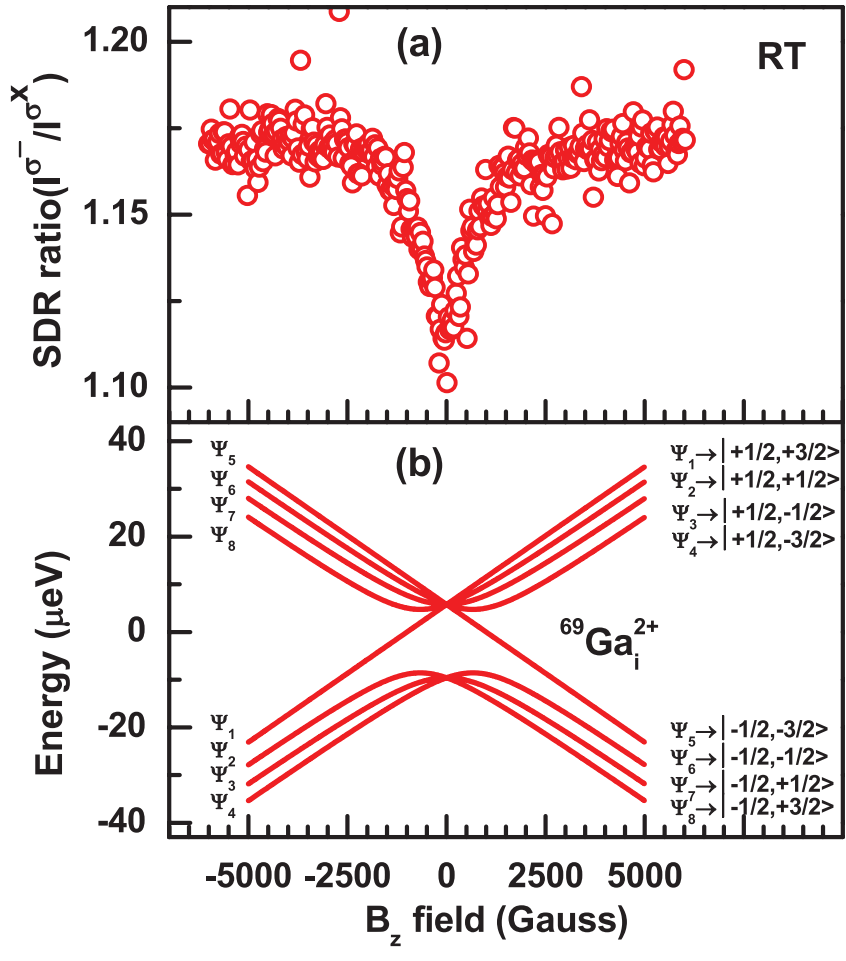

FIG. 2. (Color online) (a) Field dependence of the SDR ratio, by monitoring the peak intensity of the BB PL under optical excitation at $915 \mathrm{~nm}$ and a power of $50 \mathrm{~mW}$. (b) Calculated energy levels of the ${ }^{69} \mathrm{Ga}_{i}^{2+}$ defect from Eq. (1) with $g=2.000$ and $A=620 \times$ $10^{-4} \mathrm{~cm}^{-1}$.

the suppression of the HFI-induced spin mixing, we performed a detailed analysis of the electron spin state mixing at the $\mathrm{Ga}_{i}^{2+}$ defect with the aid of the following spin Hamiltonian:

$$
\begin{aligned}
H & =g \mu_{B} B_{z} S_{z}+\left[A S_{z} I_{z}+\frac{A}{2}\left(S_{+} I_{-}+S_{-} I_{+}\right)\right] \\
& =H_{Z}+H_{\mathrm{HF}} .
\end{aligned}
$$

Here $g$ is the electron $g$ factor of the $\mathrm{Ga}_{i}^{2+}$ defect and $\mu_{B}$ is the Bohr magnetron. The HFI term, describing the interaction between the electron spin $S$ and nuclear spin $\boldsymbol{I}$ of the defect with a hyperfine coupling constant $A$, consists of two parts. The first part involves the z projections of the defect electron and nuclear spin, $S_{z}$ and $I_{z}$, respectively. The second part, containing the raising $(+)$ and lowering $(-)$ spin operator of the electron and nucleus, plays a central role in the HFI-induced spin mixing as it mixes spin states by introducing off-diagonal elements of the spin Hamiltonian in a matrix form. The spin-filtering defect can be present in two isotopes, ${ }^{69} \mathrm{Ga}_{i}^{2+}$ and ${ }^{71} \mathrm{Ga}_{i}^{2+}$, with their natural abundance of 60.04 and $39.96 \%$, respectively. In both cases, $I=3 / 2$ and $S=1 / 2$. The hyperfine coupling constant $A$ for ${ }^{71} \mathrm{Ga}_{i}^{2+}$ is 1.27 time larger than that for ${ }^{69} \mathrm{Ga}_{i}^{2+}$, as it scales with their nuclear magnetic moments. ${ }^{18}$

By using the $g$ factor and $A$ value determined from our ODMR study (to be discussed below), the electron and nuclear spin states of the defect (taking ${ }^{69} \mathrm{Ga}_{i}^{2+}$ as an example) can be calculated by Eq. (1) and are shown in Fig. 2(b). We note that the Zeeman energies of pure electron spin states are expected to be linearly dependent on $B_{z}$ field in the absence of the HFI, according to the first term in Eq. (1). This corresponds to the case in which $H_{Z} \gg H_{\mathrm{HF}}$. In this case, the eigenstate $\psi_{n}$ of the $n$th level of the e-n spin system approaches the pure electron spin state $m_{S}$ and nuclear spin state $m_{I}$, i.e., $\psi_{n} \rightarrow\left|m_{S}, m_{I}\right\rangle$ as indicated in Fig. 2(b). Here $m_{S}$ and $m_{I}$ are the projections of the electron and nuclear spin angular momentum along the magnetic field direction, respectively, with $m_{S}= \pm 1 / 2, m_{I}= \pm 1 / 2, \pm 3 / 2$. The spin-up electron states correspond to the upper four levels when $B_{z}>5000 \mathrm{G}$, which evolve to the lower four levels when $B_{z}<-5000 \mathrm{G}$. The situation for the spin-down electron states is reversed, as indicated in Fig. 2(b). When $B_{z}$ approaches zero, the energy levels become increasingly nonlinear with $B_{z}$ that provides direct evidence for the increased mixing of the electron and nuclear spin states. At zero field, the HFI completely mixes the e-n spin, and $m_{S}$ and $m_{I}$ are no longer good quantum numbers. The resulting strongly coupled e-n system has a $J=2$ level with a fivefold degeneracy separated from a $J=1$ level with a threefold degeneracy. When the HFI-induced mixing of spin states is taken into account, the eigenstate $\psi_{n}$ of the $n$th level of the e-n spin system should be a linear combination of the pure spin states $\left|m_{S}, m_{I}\right\rangle$ in the form

$$
\begin{aligned}
\psi_{n} & =\sum_{m_{I}=-3 / 2}^{+3 / 2}\left[\sqrt{\alpha_{n m_{I}}}\left|+1 / 2, m_{I}\right\rangle+\sqrt{\beta_{n m_{I}}}\left|-1 / 2, m_{I}\right\rangle\right], \\
n & =1,2, \ldots 8 .
\end{aligned}
$$

Here, $\sqrt{\alpha_{n m_{I}}}$ and $\sqrt{\beta_{n m_{I}}}$ are the expansion coefficients of the electron spin-up and spin-down states for a given $m_{I}$, respectively. The normalization condition requires $\sum_{m_{I}=-3 / 2}^{3 / 2}\left(\alpha_{n m_{I}}+\beta_{n m_{I}}\right)=1$. It is apparent from Fig. 2 that the field range where the SDR ratio decreases is closely correlated with the mixing of the spin states induced by the HFI.

To obtain quantitative information on the spin mixing of the defect electron, we have calculated the degrees of spin mixing for all energy levels of the e-n spin system as a function of magnetic field based on their eigenstates $\psi_{n}$ obtained from Eq. (1). The probabilities of the electron spin-up and spin-down components are determined by $\sum_{m_{I}=-3 / 2}^{+3 / 2} \alpha_{n m_{I}}$ and $\sum_{m_{I}=-3 / 2}^{+3 / 2} \beta_{n m_{I}}$ and are shown by the solid and dashed curves in Fig. 3, respectively. The results shows that there exist only two energy levels that contain pure spin states, i.e., $\psi_{1}=|+1 / 2,+3 / 2\rangle$ and $\psi_{5}=|-1 / 2,-3 / 2\rangle$. This is because the spin-mixing HFI term $\frac{A}{2}\left(S_{+} I_{-}+S_{-} I_{+}\right)$has no effect on these two states. On the contrary, $\psi_{2,3,4}$ preserves only 75,50 , and $25 \%$ of the electron spin-up character at zero field that can be restored to $100 \%$ at high fields. The same conclusion can be drawn for $\psi_{8,7,6}$ but with the electron spin-down character.

As $\psi_{1,2,3,4}$ and $\psi_{5,6,7,8}$ correspond to the pure electron spin-up and spin-down state at high fields, we also trace the purity of these spin states toward zero field by calculating the averaged probabilities of the electron spin-up component $\frac{1}{4} \sum_{n=1}^{4} \sum_{m_{I}=-3 / 2}^{+3 / 2} \alpha_{n m_{I}}$ for the combined $\psi_{1,2,3,4}$ and the electron spin-down component $\frac{1}{4} \sum_{n=5}^{8} \sum_{m_{I}=-3 / 2}^{+3 / 2} \beta_{n m_{I}}$ for the combined $\psi_{5,6,7,8}$. The results for both ${ }^{69} \mathrm{Ga}_{i}^{2+}$ and ${ }^{71} \mathrm{Ga}_{i}^{2+}$ are shown in Fig. 4(b), taking as an example only the electron spin-up component. The HFI-induced electron spin mixing for ${ }^{71} \mathrm{Ga}_{i}^{2+}$ extends to a higher field as compared with that 


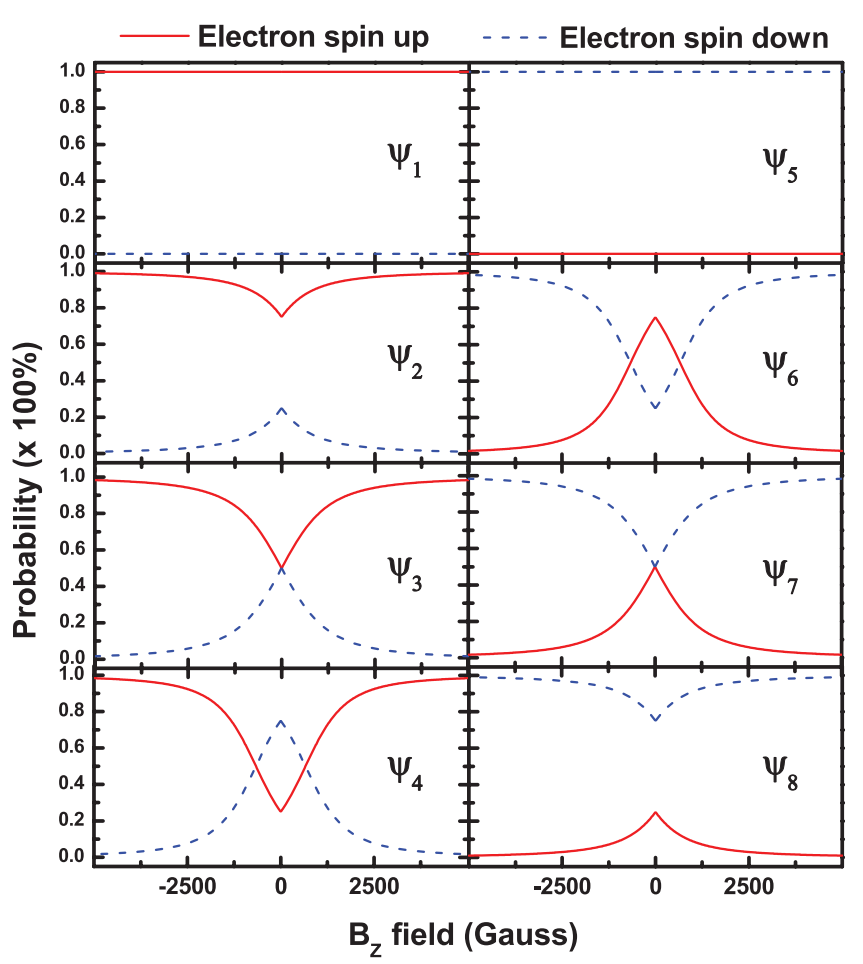

FIG. 3. (Color online) Calculated probabilities of the electron spin-up (the solid lines) and spin-down (the dashed lines) components for all eight energy levels of the ${ }^{69} \mathrm{Ga}_{i}^{2+}$ defect, with the same spin Hamiltonian parameters as used in Fig. 2(b).

for ${ }^{69} \mathrm{Ga}_{i}^{2+}$, as expected from the difference in their nuclear magnetic moment and thus the hyperfine coupling constant $A$. By this approximation, only $62.5 \%$ of the electron spin-up (or spin-down) component is preserved for $\psi_{1,2,3,4}$ (or $\psi_{5,6,7,8}$ ) at zero field, making the SDR and spin-filtering effect weaker but not completely quenched. For easier comparison, we have shown in Fig. 4(a) the calculated average degree of the electron spin mixing, including contributions from both isotopes according to their natural abundances. The field range where the mixing of the spin states is significant is found to be in excellent agreement with that where the spin-filtering effect is strongly suppressed, confirming the important role of the HFI in the spin-filtering effect. We should point out that the SDR and spin filtering via the $\mathrm{Ga}_{i}$ defects are nonlinear dynamic processes involving a coupled system of free electrons and holes, bound electrons, and nuclei. As such, we do not expect to see a linear and straightforward relation in the spin polarization between the defect and free electrons. Unfortunately, a complete quantitative analysis of the SDR and spin-filtering efficiency in a longitudinal magnetic field is not possible at present because dependences of most key parameters on magnetic fields are still unknown.

Additional evidence for the HFI-induced spin mixing of the spin-filtering defects can be obtained from an electron spin resonance (ESR) study because the spin mixing effect due to the HFI between the electron and Ga nucleus of the $\mathrm{Ga}_{i}$ defect should have a strong impact on the ESR spectrum of the defect in two ways: relative intensities and field positions of the ESR lines. Without the spin mixing, the ESR spectrum from each $\mathrm{Ga}$ isotope should consist of four lines with the
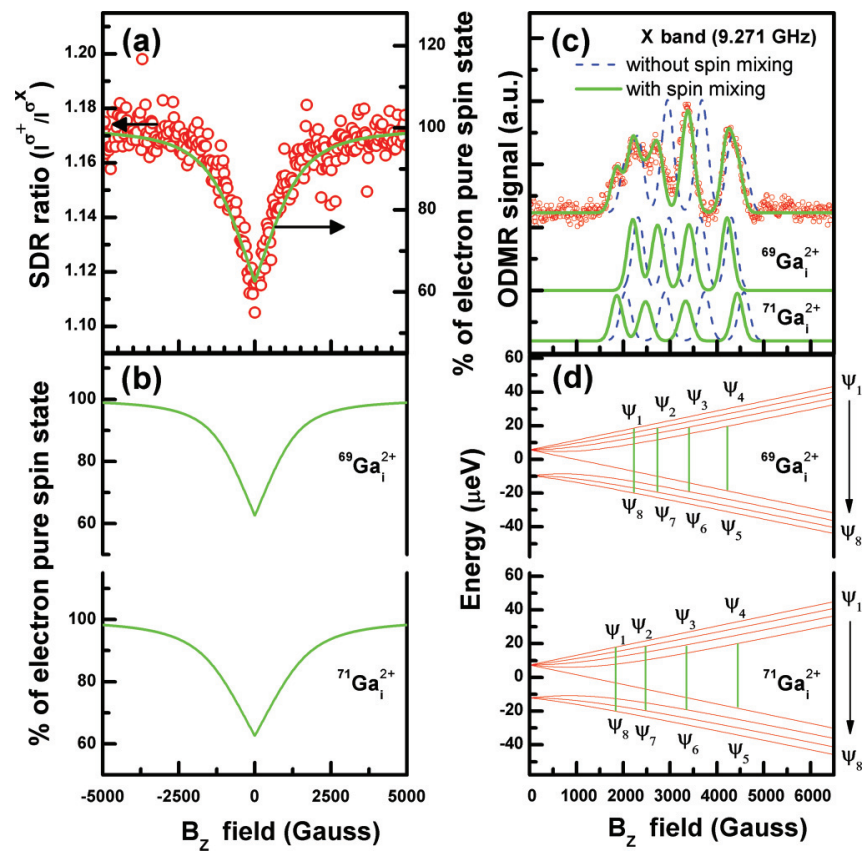

FIG. 4. (Color online) (a) Open circles: field dependence of the SDR ratio, by monitoring the peak intensity of the BB PL under optical excitation at $915 \mathrm{~nm}$ and a power of $50 \mathrm{~mW}$. Solid line: calculated and combined probability of the electron spin-up component for $\psi_{1,2,3,4}$, averaged over the contributions from both ${ }^{69} \mathrm{Ga}_{i}^{2+}$ and ${ }^{71} \mathrm{Ga}_{i}^{2+}$, taking into account their natural abundances. (b) Calculated and combined probabilities of the electron spin-up component for $\psi_{1,2,3,4}$ from ${ }^{69} \mathrm{Ga}_{i}^{2+}$ and ${ }^{71} \mathrm{Ga}_{i}^{2+}$. (c) Experimental ODMR spectrum from the defect (the open circles). The calculated ODMR spectra for the ${ }^{69} \mathrm{Ga}_{i}^{2+}$ and ${ }^{71} \mathrm{Ga}_{i}^{2+}$ isotopes based on Eq. (1) are shown by the two lower solid curves and their sum, by taking into account their natural abundance, is displayed by the solid curve overlaying with the experimental ODMR spectrum. For comparison, the corresponding ODMR spectra expected without the HFI-induced spin mixing are shown by the dashed curves. (d) Calculated eigenenergies of the ${ }^{69} \mathrm{Ga}_{i}^{2+}$ and ${ }^{71} \mathrm{Ga}_{i}^{2+}$ as a function of a longitudinal magnetic field. The vertical lines indicate the field positions of the ODMR transitions between the specified energy levels. All calculations were performed with the same spin Hamiltonian parameters as used in Fig. 2(b).

same intensity and equal spacing between the lines, as shown by the dashed lines in Fig. 4(c). As it is well known, intensity of an ESR transition is proportional to the spin-transition probability between the involved Zeeman sublevels $\psi_{n}$ and $\psi_{m}$, namely, $P_{n \leftrightarrow m} \propto\left|\left\langle\psi_{m}|V| \psi_{n}\right\rangle\right|^{2}$. Here $V$ is the magneticdipole operator containing the spin operators $S_{ \pm}$, giving rise to the ESR selection rule $\Delta m_{S}= \pm 1$ and $\Delta m_{I}=0$. Strong mixing of electron spin states is thus expected to lower the ESR transition possibility, leading to a weaker ESR signal. It also causes repelling between the interacting spin sublevels, resulting in nonlinear Zeeman splitting and nonequal distances between the ESR lines.

In the present work, ESR was performed by the ODMR technique. In ODMR, the microwave-induced change of an optical signal is used to monitor the ESR transition. ${ }^{31}$ This provides us with a highly sensitive probe for a small number of defects in the studied thin MQWs structures, which could 
not be achieved by conventional ESR techniques. A low MW frequency $(9.14 \mathrm{GHz})$ is intentionally selected here such that ESR transitions occur at low fields, where spin states are mixed, and hence the HFI-induced spin mixing can experimentally be accessed. The observed ODMR spectrum is displayed in Fig. 4(c). As expected from the ESR selection rule, four main ODMR lines corresponding to the four nuclear spin states are observed for each $\mathrm{Ga}$ isotope as illustrated in Fig. 4(d). The intensities of these four ODMR lines as well as the field separations between the ODMR lines are clearly not equal, indicating a varying degree of spin mixing between the concerned Zeeman sublevels. To examine if they originate from the same HFI as that relevant to the spin-filtering effect discussed above, we have calculated the field positions and intensities of the ODMR transitions based on the eigenenergies and eigenstates of the $\mathrm{Ga}_{i}^{2+}$ defect calculated by Eq. (1) with the same set of the spin Hamiltonian parameters used for Figs. 2(b), 3, and 4(a), 4(b). The field positions of the main ODMR transitions are indicated by the vertical lines in Fig. 4(d), together with the involved energy levels. The calculated ODMR spectra for the two isotopes of the defect are shown by the two lower solid curves in Fig. 4(c). Among the four ODMR lines from each isotope, the two outer lines are stronger than the two inner lines, which can be explained by the former involving one pure spin state $\left(\psi_{1}\right.$ or $\left.\psi_{5}\right)$ as the initial or final state of the ESR transition, whereas for the latter, both the initial and final state of the ESR transitions are spin mixed. The simulated ODMR spectrum including the contributions from both $\mathrm{Ga}$ isotopes is depicted by the solid line in the upper part of Fig. 4(c), in excellent agreement with the experimental data. Our ODMR results have therefore provided convincing evidence that the spin-mixing effect due to the central HFI is still active over the ODMR field range (2000-4500 G), although much weakened as compared with that near zero field. This is consistent with the calculated spin mixing shown in Fig. 4(b) and the experimental SDR data in Fig. 4(a), which show a slow varying but nonsaturation behavior over the same field range. We also performed a comparative ODMR study at a higher microwave frequency $(34 \mathrm{GHz})$ and thus at higher fields (above $9000 \mathrm{G}$; not shown here), where the ODMR lines show nearly equal intensity as expected from the suppression of the spin mixing by the HFI.

\section{CONCLUSION}

From the magneto-optical and ODMR studies, we have provided direct and compelling experimental evidence that the HFI of the spin-filtering $\mathrm{Ga}_{i}$ defects causes a strong mixing of the e-n spin states of the defects and thereby degrades the efficiency of the RT spin-blockade and spin-filtering effect in GaNAs. This conclusion is firmly supported by our theoretical calculations of the spin mixing from a detailed spin Hamiltonian analysis. This work thus stresses the importance as well as provides a guideline to suppress or avoid the HFI occurring at the spin-filtering defects. One strategy to further improve the spin-filtering efficiency is to seek for alternative spin-filtering defects with zero nuclear spin or weak hyperfine coupling. Even in terms of the currently employed spinfiltering $\mathrm{Ga}_{i}$ defects, it is known from our earlier studies that they can be present in $\mathrm{Ga}$ (In)NAs in many forms with different HFI strengths determined by the localization of electron wave function at the defect core. ${ }^{22}$ The formation of various $\mathrm{Ga}_{i}$ interstitial defects with different configurations strongly depends on growth conditions and postgrowth treatments, providing a possibility to engineer hyperfine coupling and thus the spin-filtering efficiency. Further studies along this line are currently underway.

Our results also suggest that the effect of the HFI-induced spin mixing, thus far neglected in early studies, should be properly considered in rate equation analyses in order to yield an accurate description of the defect-engineered spin-filtering effect.

\section{ACKNOWLEDGMENTS}

We are grateful to $\mathrm{C}$. $\mathrm{W}$. Tu for providing the samples. This work was supported by Linköping University through the professor contracts, Swedish Research Council (Grant No. 621-2011-4254), Swedish Energy Agency, and the Knut and Alice Wallenberg Foundation.
${ }^{1}$ Semiconductor Spintronics and Quantum Computation, edited by D. Awschalom, D. Loss, and N. Samarth (Springer Verlag, Berlin, 2002).

${ }^{2}$ Spin Physics in Semiconductors, edited by M. I. Dyakonov (Springer, Berlin, 2008).

${ }^{3}$ Handbook of Spintronic Semiconductors, edited by W. M. Chen and I. A. Buyanova (Pan Stanford, Singapore, 2010).

${ }^{4}$ S. I. Erlingsson, Y. V. Nazarov, and V. I. Fal'ko, Phys. Rev. B 64, 195306 (2001).

${ }^{5}$ A. V. Khaetskii, D. Loss, and L. Glazman, Phys. Rev. Lett. 88, 186802 (2002).

${ }^{6}$ R. de Sousa and S. Das Sarma, Phys. Rev. B 68, 115322 (2003).

${ }^{7}$ L. Childress, M. V. Gurudev Dutt, J. M. Taylor, A. S. Zibrov, F. Jelezko, J. Wrachtrup, P. R. Hemmer, and M. D. Lukin, Science 314, 281 (2006).
${ }^{8}$ M. V. Gurudev Dutt, L. Childress, L. Jiang, E. Togan, J. Maze, F. Jelezko, A. S. Zibrov, P. R. Hemmer, and M. D. Lukin, Science 316, 1312 (2007).

${ }^{9}$ A. S. Bracker, E. A. Stinaff, D. Gammon, M. E. Ware, J. G. Tischler, A. Shabaev, Al. L. Efros, D. Park, D. Gershoni, V. L. Korenev, and I. A. Merkulov, Phy. Rev. Lett. 94, 047402 (2005).

${ }^{10}$ J. R. Petta, A. C. Johnson, J. M. Taylor, E. A. Laird, A. Yacoby, M. D. Lukin, C. M. Marcus, M. P. Hanson, and A. C. Gossard, Science 309, 2180 (2005).

${ }^{11}$ A. C. Johnson, J. R. Petta, J. M. Taylor, A. Yacoby, M. D. Lukin, C. M. Marcus, M. P. Hanson, and A. C. Gossard, Nature (London) 436, 925 (2005).

${ }^{12}$ I. A. Merkulov, A. L. Efros, and M. Rosen, Phys. Rev. B 65, 205309 (2002).

${ }^{13}$ W. A. Coish and D. Loss, Phys. Rev. B 70, 195340 (2004). 
${ }^{14}$ M. I. Dyakonov and V. I. Perel', Sov. Phys. JETP 36, 995 (1973).

${ }^{15}$ Y. Sheng, T. D. Nguyen, G. Veeraraghavan, Ö. Mermer, M. Wohlgenannt, S. Qiu, and U. Scherf, Phys. Rev. B 74, 045213 (2006).

${ }^{16}$ J. Beyer, Y. Puttisong, I. A. Buyanova, S. Suraprapapich, C. W. Tu, and W. M. Chen, Appl. Phys. Lett. 100, 143105 (2012).

${ }^{17}$ X. J. Wang, I. A. Buyanova, and W. M. Chen, Phys. Rev. B 86, 205202 (2012).

${ }^{18}$ X. J. Wang, I. A. Buyanova, F. Chao, D. Zhao, D. Lagarde, A. Balocchi, X. Maries, C. W. Tu, J. C. Harmand, and W. M. Chen, Nat. Mater. 8, 198 (2009).

${ }^{19}$ V. K. Kalevich, E. L. Ivchenko, M. M. Afanasiev, A. Yu. Shiryaev, A. Yu. Egorov, V. M. Ustinov, and Y. Masumoto, JETP Lett. 82, 455 (2005).

${ }^{20}$ V. K. Kalevich, A. Yu. Shiryaev, E. L. Ivchenko, A. Yu. Egorov, L. Lombez, D. Lagarde, X. Marie, and T. Amand, JETP Lett. 85, 174 (2007).

${ }^{21}$ D. Lagarde, L. Lombez, X. Marie, A. Balocchi, T. Amand, V. K. Kalevich, A. Shiryaev, E. Ivechenko, and A. Egorov, Phys. Status Solidi A 204, 208 (2007).
${ }^{22}$ X. J. Wang, Y. Puttisong, C. W. Tu, A. J. Ptak, V. K. Kalevich, A. Yu. Egorov, L. Geelhaar, H. Riechert, W. M. Chen, and I. A. Buyanova, Appl. Phys. Lett. 95, 241904 (2009).

${ }^{23}$ Y. Puttisong, X. J. Wang, I. A. Buyanova, H. Carrére, F. Zhao, A. Balocchi, X. Marie, C. W. Tu, and W. M. Chen, Appl. Phys. Lett. 96, 052104 (2010).

${ }^{24}$ Y. Puttisong, X. J. Wang, I. A. Buyanova, C. W. Tu, L. Geelhaar, H. Riechert, and W. M. Chen, Appl. Phys. Lett. 98, 012112 (2011).

${ }^{25}$ Y. Puttisong, D. Dagnelund, I. A. Buyanova, C. W. Tu, A. Polimeni, M. Capizzi, and W. M. Chen, Appl. Phys. Lett. 99, 152109 (2011).

${ }^{26}$ E. L. Ivchenko, V. K. Kalevich, A. Yu. Shiryaev, M. M. Afanasiev, and Y. Masumoto, J. Phys.: Condens. Matter 22, 465804 (2010).

${ }^{27}$ A. Kunold, A. Balocchi, F. Zhao, T. Amand, N. Ben Abdallah, J. C. Harmand, and X. Marie, Phys. Rev. B 83, 165202 (2011).

${ }^{28}$ V. K. Kalevich, M. M. Afanasiev, A. Yu. Shiryaev, and A. Yu. Egorov, Phys. Rev. B 85, 035205 (2012).

${ }^{29}$ Y. Puttisong, I. A. Buyanova, A. J. Ptak, C. W. Tu, L. Geelhaar, H. Riechert, and W. M. Chen, Adv. Mater. 25, 738 (2013).

${ }^{30}$ F. Meier and B. P. Zakharchenya, Optical Orientation (NorthHolland, Amsterdam, 1984).

${ }^{31}$ For a review on ODMR, see, e.g., W. M. Chen, Thin Solid Films 364, 45 (2000). 